\title{
El Gasto Energético del Venado Cola Blanca (Odocoileus virginianus texanus) en Relación a la Precipitación en una Zona Semiárida de México
}

\author{
Sonia Gallina ${ }^{1 *}$ y Joaquin Bello ${ }^{2}$
}

\section{Resumen}

Los requerimientos energéticos de un animal dependen de su metabolismo basal, actividad y estado fisiológico. Se desconoce como es el gasto energético del venado cola blanca texano en zonas áridas y semiáridas. Por lo tanto es importante conocer como responden los individuos a los cambios en su ambiente, derivados principalmente de los cambios por la precipitación a lo largo del tiempo que afecta al alimento y la cobertura. Se analiza el gasto energético del venado cola blanca texano y su variación de acuerdo al tipo de comportamiento, sexo, época y año en el noreste de México. Este trabajo fue realizado en el Rancho San Francisco y en el Rancho Peñitas, en los Municipios de Lampazos, Nuevo León y Progreso, Coahuila, México. Se utilizó radiotelemetría siguiendo 10 machos y 14 hembras de venado (con collares con sensor de actividad) desde 1995 a 1998. Se pudieron registrar tres actividades principales: alimentación, desplazamiento y descanso. Se encontraron diferencias significativas entre las tres pautas de comportamiento, el mayor gasto ocurre con el descanso. La interacción época-año, y entre sexos solamente hubo diferencias en la pauta de alimentación. El gasto energético del venado cola blanca en zonas áridas si difiere entre sexos ya que los machos gastaron más energía (1726 \pm 38 kcal/día/individuo) que las hembras (1556 \pm 35 $\mathrm{kcal} /$ día/individuo). Los venados tuvieron un menor gasto energético para alimentarse y moverse en los años con condiciones de sequía más severa como 1995 y 1996, cuando el alimento es más escaso, lo que indica que los venados en estas zonas áridas tienen una estrategia conductual para tratar de ahorrar energía cuando las condiciones ambientales son desfavorables. Aunque en años secos como 1995 los venados gastan energía para actividades fundamentales como es la reproducción.

Palabras Clave: energética, época fisiológica, metabolismo, pautas de actividad, radiotelemetría, México.

\footnotetext{
1*Instituto de Ecología A.C. Carretera Antigua a Coatepec No. 351. AP 63. Xalapa 91070 Veracruz México. E-mail: sonia. gallina@inecol.edu.mx, autor corresponsal

${ }^{2}$ División Académica de Ciencias Biológicas, Universidad Juárez Autónoma de Tabasco, km 0.5 Carretera Villahermosa Cárdenas, Villahermosa,Tabasco,CP 86039, E-mail: joaquin.bello@cicea.ujat.mx
} 
The energetic requirements of animals are dependent upon their basal metabolism, behavior and physiological conditions. Every activity of the animal represents an energetic cost. It is unknown how deer deal with the thermal conditions in arid and semiarid areas where the air temperature is higher than $40^{\circ} \mathrm{C}$ and the precipitation is lower than $400 \mathrm{~mm}$ annually. Therefore, it is important to understand the energetic cost between sexes under different physiological conditions and in different years. This can also help us understand the response of individuals to annual variation in environmental changes such as the amount of precipitation that influences food availability and amount of protective cover. This research was carried out in Rancho San Francisco and Rancho Peñitas, in the Municipality of Lampazos, Nuevo León and Progreso, Coahuila, México. From 1995 to 1998, 10 bucks and 14 does with radiocollars (with activity sensor) were tracked. We were able to record three main activities: feeding, walking and resting. We found significant differences in each of the three activity patterns and found that the greatest energy expenditure occurs while the animal is resting.

The energy expenditure of the white tailed deer in arid zones differs between sexes because males spent more energy $(1726 \pm 38 \mathrm{kcal} /$ day/individual $)$ than females (1556 $\pm 35 \mathrm{kcal} /$ day/individual). We found significant differences between season-year interactions in the three activity patterns.

However, the only difference we found between sexes was the energy expenditure while feeding. Deer in arid zones had less energetic expenditure in years with severe drought, when food was scarce, suggesting that they are capable of modifying their behavioral strategies in order to save energy when environmental conditions are unfavorable.

Key words: energetic, physiological condition, metabolism, activity pattern, radiotracking, Mexico.

Todos los organismos deben utilizar energía para mantener sus funciones vitales. La conversión de energía de una forma a otra en los organismos es llamado metabolismo. El metabolismo basal ha sido definido como el costo energético mínimo cuando un animal está en reposo, en un ambiente termoneutral (Brody 1945 citado por Moen 1973). Crampton y Harris (1969, cit. Moen 1973) estimaron que el $75 \%$ de la energía del metabolismo basal se gasta en mantener el tono muscular y la temperatura corporal, y el $25 \%$ es usado para la circulación, excreción, secreción y respiración.

Los requerimientos energéticos dependen del metabolismo basal, la actividad y el estado fisiológico del organismo (Moen 1978). Cualquier actividad del individuo representa un "costo" en términos de energía. Los animales responden conductualmente al régimen térmico, alterando el balance entre la pérdida de calor y la producción de calor, mediante cambios en la orientación, postura, actividad o selección de cobertura para protección (Wallmo 1981).

Los requerimientos energéticos pueden calcularse para las distintas actividades, y la suma será el requerimiento de energía total diario. Así, el balance entre los costos 
energéticos (ganancia menos pérdida), definitivamente influirá en las posibilidades de sobrevivencia y reproducción de los individuos, y por lo tanto, en la tasa de crecimiento de la población. Para las hembras de venado cola blanca el último tercio de la gestación (que en este caso coincide con la época seca) es el de mayor metabolismo energético (Kroll 1992). Según Pekins et al. (1998) el requerimiento de energía durante esta etapa es 30 a 45\% superior a las necesidades de mantenimiento, y así también los requerimientos energéticos durante la lactancia pueden alcanzar 2.3 veces la tasa metabólica basal (Wallmo 1981). Mientras que para los venados machos, es la época de apareamiento la que implica un aumento en el gasto energético, ya que tienen que defender a las hembras en estro, contra otros machos, para asegurar su descendencia. Durante este periodo de celo los machos pueden perder incluso hasta el 30\% del peso corporal ya que prácticamente dejan de alimentarse (Kroll y Koerth 1966; Villarreal 1999).

El gasto energético de cualquier animal dependerá de las condiciones intrínsecas del individuo (estado fisiológico, sexo, edad) y de las condiciones extrínsecas como el tipo de vegetación, cantidad y calidad de las plantas disponibles como forraje, cobertura de protección, de la disponibilidad de agua libre, de las características de temperatura, humedad y precipitación del ambiente (Ockenfels y Bissonette 1984; Verme y Ullrey 1984; Beier y McCullough 1990; Ockenfels et al. 1991; Kroll 1992). Moen (1978) encontró que el metabolismo más bajo en el venado cola blanca ocurre en invierno y el más alto en verano, siendo este ritmo una adaptación para conservar energía, la necesidad de recursos forrajeros es menor cuando los recursos disponibles son reducidos. Además encontró que el mayor gasto energético fue para una hembra con dos crías durante el período de lactancia y el menor gasto (1.5 veces el metabolismo basal) en invierno (época reproductiva). Aunque Mautz et al. (1992) examinan la tasa metabólica de hembras de venado cola blanca cautivas (en animales echados, es decir en descanso, y dentro de la zona termal neutral) no encontraron diferencias significativas entre estaciones, señalando que tal vez los cambios encontrados en otros estudios, con un incremento de la tasa metabólica en verano, pudieron deberse a la medida de la tasa metabólica fuera de la zona termoneutral. El comportamiento es importante porque voluntariamente el venado en general, puede evitar circunstancias desagradables del ambiente, como el intenso calor o el frío y los vientos (Wallmo 1981). Estos cambios conductuales se han estudiado también en el matorral xerófilo del Noreste de México (Bello et al. 1996, 1998, 2001, 2003a, 2003b, 2004, Corona-Zárate 1999, Delfín et al. 1998, Gallina et al. 1998, Gallina y Bello 2002, Gallina et al. 2003).

Muchas especies de mamíferos en ambientes áridos tienen requerimientos metabólicos que son menores a los esperados por su tamaño corporal (Hudson y Christopherson 1985, Lovegrove 2000). Las especies de zonas áridas de productividad primaria baja tienen tasas metabólicas menores que sus congéneres de zonas templadas con mayor productividad (Mueller y Diamond 2001 cit Strickland et al. 2005). Por lo tanto, según Strickland et al. (2005) el efecto de diferentes condiciones ambientales (temperatura, precipitación) sobre la termorregulación del animal, y sobre la disponibilidad y calidad del forraje, puede influir en los requerimientos metabólicos y en los requerimientos energéticos para mantener la masa corporal. Los menores requerimientos de manutención pueden ser una respuesta adaptativa a ambientes de baja productividad. Las menores tasas metabólicas son encontradas en especies adaptadas a 
desiertos que subsisten de forrajes de baja calidad (Strickland et al. 2005).

Se desconoce como se enfrenta energéticamente el venado cola blanca en zonas áridas y semiáridas, donde las temperaturas rebasan los $40^{\circ} \mathrm{C}$, y las precipitaciones no llegan a $400 \mathrm{~mm}$ anuales, ya que el límite termoneutral de esta especie de venado son los $30^{\circ} \mathrm{C}$ (Ockenfels y Bissonette 1984), y a temperaturas superiores empieza a perder agua por transpiración. Por lo tanto es importante, conocer el gasto energético de los dos sexos en los diferentes estados fisiológicos (si están en época reproductiva, posreproductiva o de gestación o en época prereproductiva o de crianza,), y en diferentes años, especialmente para entender como responden los individuos a los cambios en su ambiente. Estos cambios, derivados principalmente de la diferencia en la cantidad y distribución de la precipitación a lo largo del tiempo, tienen una influencia en la calidad del hábitat, al afectar directamente la vegetación, es decir, en la cantidad y calidad del alimento disponible, así como en la cobertura de protección.

El objetivo del estudio fue analizar el gasto energético del venado cola blanca texano (Odocoileus virginianus texanus) en un matorral xerófilo del Noreste de México y la influencia que tiene las condiciones fisiológicas y las ambientales como la precipitación. Conocer si existen diferencias entre los sexos, las épocas y años con distintos niveles de precipitación, así como también ver si existe correlación del gasto energético con la precipitación.

Las hipótesis de trabajo son las siguientes: 1) esperamos que las hembras tengan un mayor gasto energético total durante la época de crianza, mientras que los machos lo tengan durante la época de celo o reproductiva. 2) Esperamos diferencias en cuanto al gasto energético por actividad de acuerdo a las condiciones ambientales, siendo menor el gasto en cuanto a las pautas de alimentación y desplazamiento durante la escasees de alimento y agua (época posreproductiva que coincide con la época seca del año), ya que deberán ahorrar energía que no puede ser suplida fácilmente dadas las condiciones ambientales. 3) Durante los años de mayor precipitación el gasto energético deberá ser mayor ya que pueden buscar mejor alimento y en mayor cantidad, compensando el gasto por obtener alimento de mejor calidad.

Área de Estudio.- Este trabajo fue realizado en el Rancho San Francisco, entonces propiedad de DUMAC, localizado entre los Municipios de Lampazos, Nuevo León y

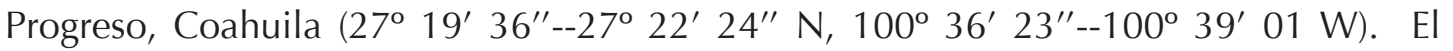
rancho tiene una superficie de 1000 ha cercada con malla venadera, con 32 bebederos artificiales y tres, donde se estima una población de venados entre 80 y 100 individuos. También se realizó parte del trabajo en el Rancho Peñitas, con una extensión de 11000 ha, localizado anexo al Rancho San Francisco. El clima en la región es semi-seco, con precipitación promedio anual menor a $400 \mathrm{~mm}$ y temperatura promedio anual de $21^{\circ} \mathrm{C}$, con una época de lluvia de mayo a septiembre, que presenta gran variabilidad interanual (Bello et al. 2004). La vegetación de la zona corresponde a un matorral xerófilo, con alta riqueza de especies ya que se mezclan dos provincias fisiográficas: la Llanura costera del Golfo Norte y la Gran Llanura de Norteamérica (Briones 1984). Existen siete asociaciones vegetales: pastizal de Hilaria (3\%), nopalera de Opuntia (1\%), matorral 
de cenizo Leucophyllum frutescens (11\%), matorral de hojasen Flourensia cernua (6\%), matorral de Acacia-Prosopis (54\%), matorral de mezquite Prosopis (15\%) y matorral de Acacia-Celtis (10\%).

Métodos.- Se capturaron y pesaron 10 machos y 14 hembras adultas de venado cola blanca desde septiembre de 1994, con redes de caída, para colocarles collares con sensor de actividad modelo 400 (con fecuencias de 150- $152(\mathrm{MHz})$ marca TELONICS (Inc., Mesa, Arizona). Estos collares fueron probados primero con venados en cautiverio, y se encontró que se pueden categorizar tres pautas de comportamiento de acuerdo al número de pulsaciones (beeps) por minuto: echado o descansando (50-55 pulsos por minuto), locomoción o movimiento (56-69 pulsos por minuto), y alimentación (70-87 pulsos por minuto, ver detalles en Mandujano et al. 1996). Los venados fueron pesados con un dinamómetro marca PESOLA de $100 \mathrm{~kg}$. Los conteos se hicieron de 5-7 minutos por individuo, y se registraban todos los animales cada hora por ciclos de 24 horas, mediante un equipo de recepción TR-2 y TR-4 marca (Telonics), utilizando antenas $\mathrm{H}$, y antenas fijas Yagi paralelas de 4 elementos cada una, Ilevando a cabo 2-3 ciclos al mes para cada venado de 1995 a 1998. El número de animales que se seguían varió en las épocas y años (por mortalidad y captura).

Se consideraron tres etapas del ciclo de vida según el estado fisiológico para los muestreos: reproductivo o de celo (noviembre-febrero), ocurre cuando las hembras entran en estro y los machos andan en su búsqueda para aparearse y pueden ocurrir las peleas entre machos cuando encuentran hembras en estro; posreproductivo o de gestación (marzo a junio) durante el cuál los machos recuperan peso, y pierden las astas, mientras las hembras están gestantes (dura siete meses la gestación) y de crianza o prereproductiva (julio-octubre), cuando las hembras están lactantes y los machos, con sus astas creciendo, se preparan para la época reproductiva, y coincide con la época de Iluvias cuando el alimento es abundante y de mayor calidad (Villarreal 1999, Bello et al. 2001).

El metabolismo basal se obtuvo para cada individuo con la fórmula $\mathbf{Q}_{\mathbf{m b}}=\mathbf{c W}^{\mathbf{b}}$ donde $\mathbf{Q}_{\mathbf{m b}}$ es la energía gastada en el metabolismo basal, $\mathbf{c}=$ es una constante (tiene un valor de $70 \mathrm{kcal} / \mathrm{kg}$ ), W es el peso del animal y el exponente $\mathbf{b}=0.75$ (valor adoptado por el National Research Council 1966 cit. por Moen 1973). Se asumió que el peso obtenido cuando se capturaron los individuos como constante, aunque sabemos que varía con los años y también con las épocas y condiciones del individuo.

El valor expresado como metabolismo basal, se utilizó como medida correspondiente a la categoría o pauta de descanso y podría estar sobrevaluado ya que el metabolismo basal se realiza a temperatura constante, lo cual no ocurre en estado silvestre, sin embargo puede considerarse como un buen indicador del gasto energético (Moen 1978). Para la pauta de movimiento se multiplicó por 1.64, ya que el terreno es prácticamente plano, y para la categoría de alimentación por 1.59 (Moen 1973). Para obtener el gasto energético por día (kcal/día), se consideró la proporción de tiempo dedicada a cada actividad con su respectivo costo metabólico.

Se aplicó un ANOVA de tres vías para cada una de las pautas de comportamiento para conocer si había diferencias en el tiempo invertido entre sexos, épocas y años. También se utilizaron ANOVAs de tres vías para comparar los datos del gasto energético 
de cada categoría de comportamiento, de acuerdo al estado fisiológico (reproductivo, posreproductivo y prereproductivo o crianza), sexo y año, además de sus interacciones (1995, 1996, 1997, 1998; Zar 1996). La Prueba de Tukey a posteriori se aplicó para cada variable para conocer donde variaban significativamente los gastos energéticos. La precipitación se analizó mensualmente considerando los cuatro años de estudio calculando el coeficiente de variación por época y por año, para conocer qué tan variable es la cantidad de lluvia y analizar en función de ello el gasto energético de los venados. Se hicieron correlaciones entre la precipitación y el gasto energético (separando sexos y pautas). Para conocer si el gasto energético se relaciona con la precipitación se analizó primero la variación de esta última entre las diferentes épocas por año mediante el uso del coeficiente de variación, debido a que la cantidad y distribución de la precipitación a lo largo del año puede influir en el comportamiento del venado (Bello et al. 2003a, 2003b, Gallina et al. 2003, Bello et al. 2004).

\section{Resultados}

Durante los cuatro años de estudio (1995-1998), se analizaron en total 24 ciclos diarios de actividad (promedios de 2-3 ciclos individuales completos de cada mes) de machos y 34 ciclos diarios de actividad de hembras.

Pautas de comportamiento.- Se encontró que el mayor tiempo (aproximadamente el $60 \%$ ) lo pasan echados (descansando o rumiando), el $16 \%$ alimentandose y el resto del tiempo lo usan para desplazarse. Se encontraron diferencias significativas en las tres pautas por año y la interacción época-año (Tabla 1), solamente la pauta de movimiento fue diferente entre los sexos al igual que la de alimentarse, entre épocas (Tabla 1).

\begin{tabular}{ccccc}
\hline Comportamiento & $\begin{array}{c}\text { Fuente de } \\
\text { variación }\end{array}$ & GL & F & $P$ \\
\hline \multirow{2}{*}{ Echado } & Año & 3,187 & 12.8 & $<0.001$ \\
& Época x año & 6,187 & 6.3 & $<0.001$ \\
Movimiento & Sexo & 1,187 & 7.3 & 0.008 \\
& Año & 3,187 & 12.9 & $<0.001$ \\
& Sexo X año & 3,187 & 2.7 & $<0.047$ \\
& Época X año & 6,187 & 5 & $<01$ \\
& Época & 2,187 & 8.5 & $<0.001$ \\
& Año & 3,187 & 7.3 & $<0.001$ \\
& Epoca X año & 6,187 & 2.2 & 0.042 \\
\hline
\end{tabular}

Tabla 1. Valores significativos de los ANOVAS realizados para cada pauta de comportamiento y sus fuentes de variación (GL: Grados de libertad, F: Valor de F de Fisher, P: Probabilidad).

Gastos energéticos totales.- Se encontraron diferencias significativas entre sexos en cuanto al gasto total $\left(F_{1,58}=10.95, P=0.002\right)$. Los machos gastaron más energía (1726 $\pm 38 \mathrm{kcal} /$ día/individuo) que las hembras (1556 $\pm 35 \mathrm{kcal} /$ día/individuo). Entre años también se encontraron diferencias significativas $\left(F_{3,58}=13.61, P<0.001\right)$, siendo menor el gasto energético en el año 1995 (1462 \pm 51 kcal/día/individuo) que fue el año más seco (sólo $135.5 \mathrm{~mm}$ ), en comparación con los gastos mayores en 1997 (1755 $\pm 44 \mathrm{kcal} /$ día/individuo) que fue el año en que cayó más precipitación (357mm), y 1996 (1724 I $42 \mathrm{kcal} / \mathrm{dí} /$ individuo). 
Gastos energéticos por tipo de actividad.- Se encontraron diferencias significativas en el gasto energético por tipo de actividad $\left(\mathrm{F}_{2,222}=58.8, P<0.001\right)$, la actividad que tuvo mayor gasto energético fue descansar, ya que es a la que dedican más de su tiempo y la de menor gasto fue la de alimentación (Fig. 1).

$\mathrm{Al}$ analizar por tipo de actividad, para la pauta descanso, se encontraron diferencias significativas entre sexos (Tabla 2): los machos gastan más energía que las hembras porque permanecen más tiempo echados. Entre épocas se encontraron diferencias significativas, ya que en la época posreproductiva fue menor el gasto energético en comparación con las otras dos épocas (Fig. 2). En cuanto a las diferencias entre años también fueron significativas (Tabla 2), el gasto energético fue mayor en 1998 en comparación con los dos primeros años, además el gasto de 1997 fue mayor que el de 1995 (Fig. 1).

Para la actividad de desplazamiento o movimiento, los venados gastaron significativamente menos energía (Tabla 2) en 1996 en comparación con los otros tres años. Además se encontraron diferencias significativas en la interacción época por año, siendo durante la época de celo o reproductiva cuando ocurre un mayor gasto en comparación con las otras dos épocas en 1995, sin embargo, para 1996 se encontró que el gasto energético fue mayor en la época de crianza (Fig. 3).

Con la actividad de alimentación se encontraron diferencias significativas entre épocas, años y la interacción época-año (Tabla 2). Durante la época posreproductiva el gasto energético es menor para alimentarse en comparación con las otras dos épocas (Fig. 4). Entre años el menor gasto fue durante 1995 en comparación con 1997 y 1998. Para la interacción época-año para la época posreproductiva en 1996 y 1997 el gasto energético fue mayor en comparación con 1995 (Fig. 4).

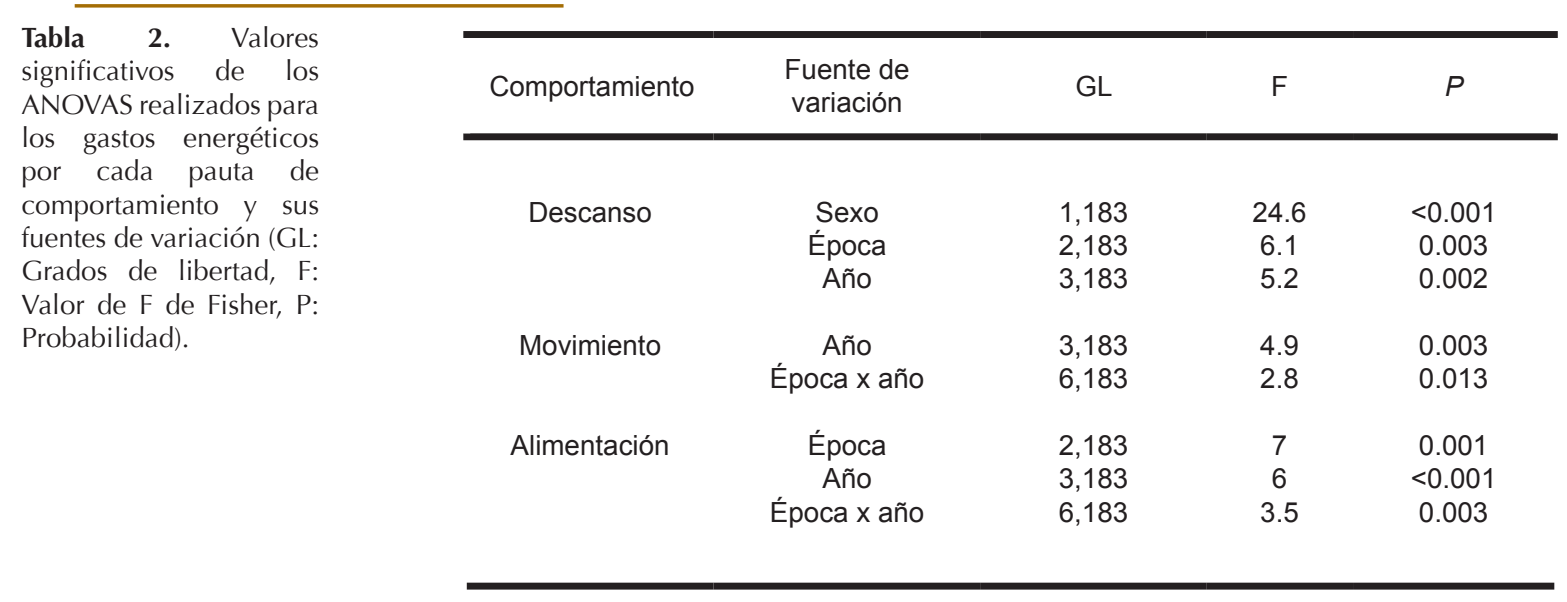

Variación de la precipitación y su relación con el gasto energético.- Al analizar la precipitación de acuerdo a las épocas fisiológicas (Fig. 5), no se encontraron diferencias significativas $\left(F_{2,9}=3.37, P=0.08\right)$, entre épocas, lo cual tal vez se deba a la alta variación en la precipitación por época entre años y al bajo tamaño de muestra, que afectó la potencia de la prueba (0.352). No se encontraron diferencias entre años, en la precipitación durante la época reproductiva o de celo en los cuatro años, pero es cuando ocurre la menor precipitación así como variación (promedio 31.88 mm, CV = 12.04, 


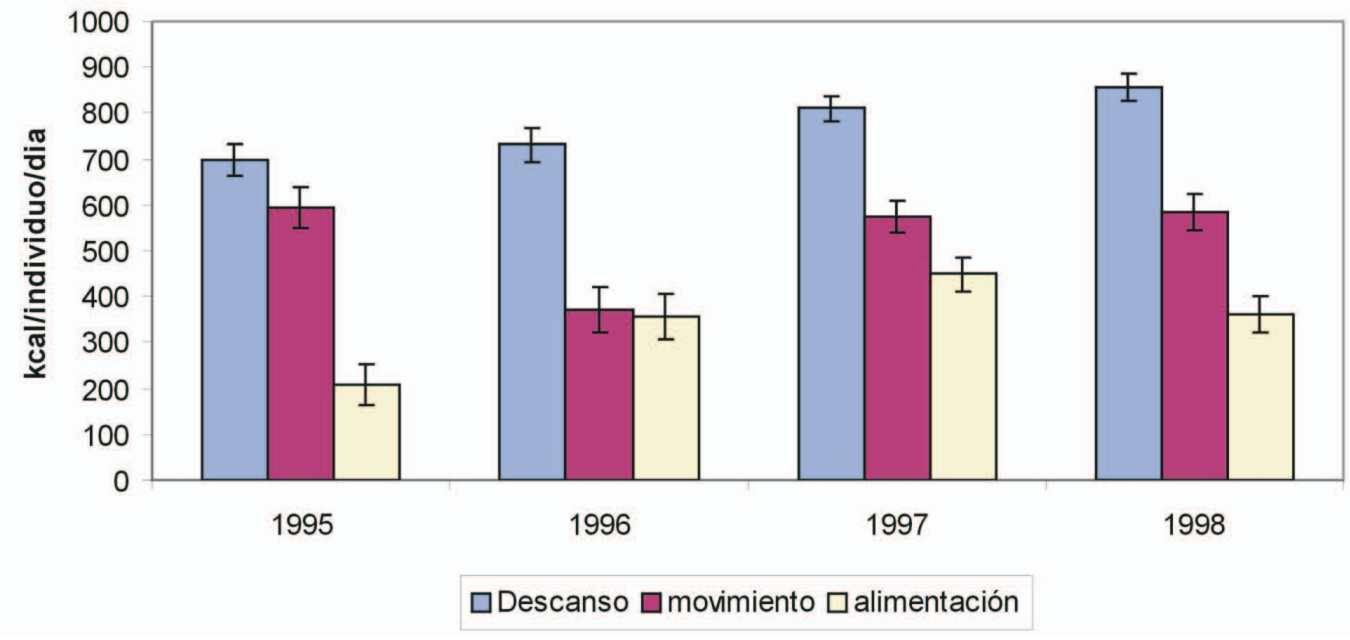

Figura 1. Gasto energético (kcal/día/ individuo) por tipo de actividad para el venado cola blanca en los diferentes años en un matorral xerófilo del Noreste de México.

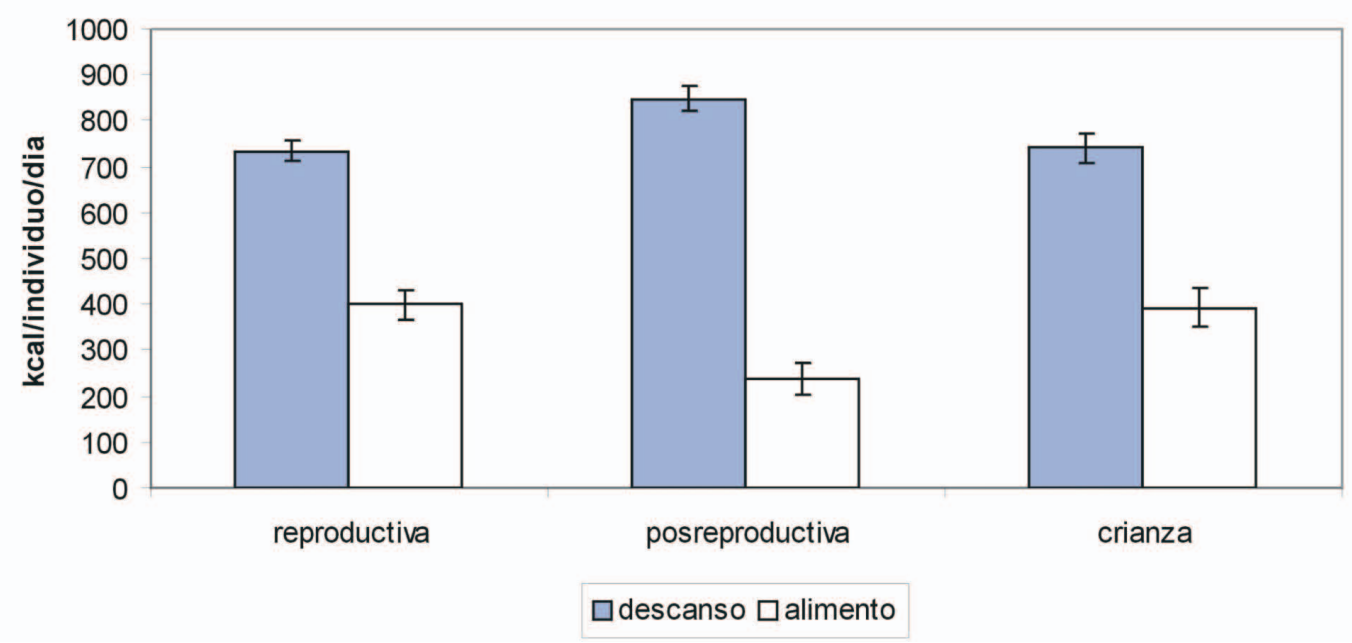

Figura 2. Gasto energético ( $\mathrm{kcal} / \mathrm{día}$ individuo) de la pauta descanso y alimentación en las tres épocas fisiológicas del venado cola blanca en un matorral xerófilo del Noreste de México.

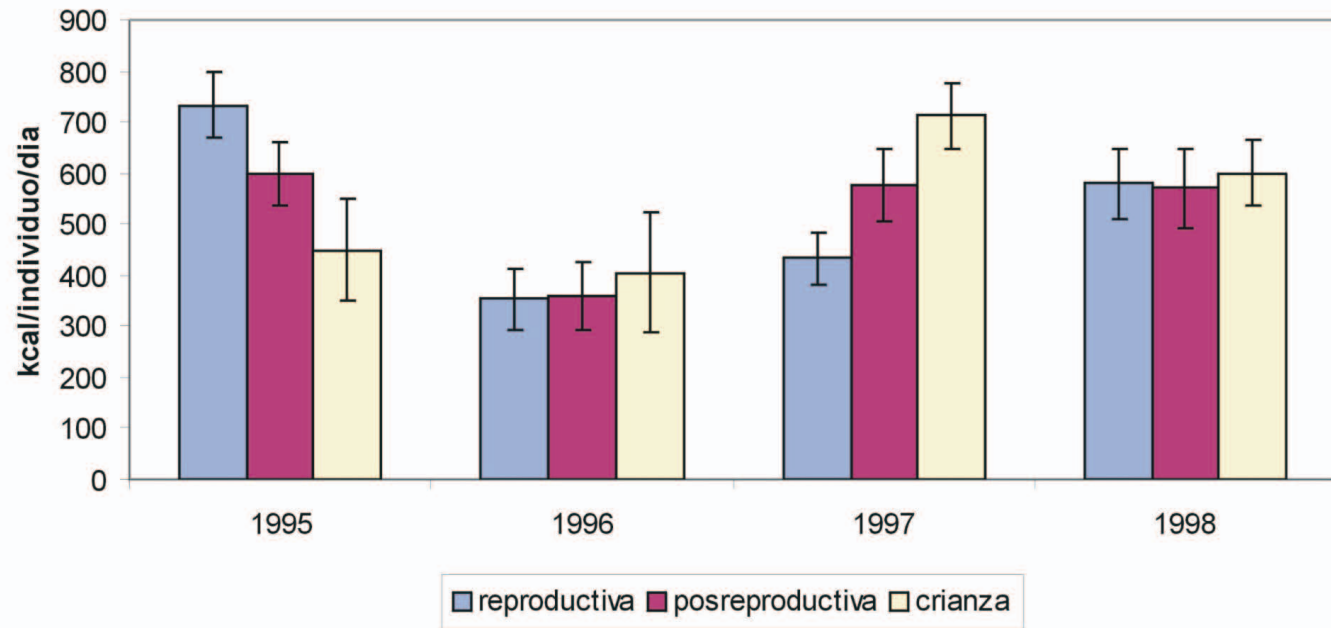

Figura 3. Gasto energético del desplazamiento en las diferentes épocas fisiológicas y años para el venado cola blanca en un matorral xerófilo del Noreste de México. 
Figura 4. Gasto energético en cuanto a la alimentación en las diferentes épocas y años para el venado cola blanca en un matorral xerófilo del Noreste de México.
Figura 5. Precipitación por época fisiológica durante los cuatro años de estudio.
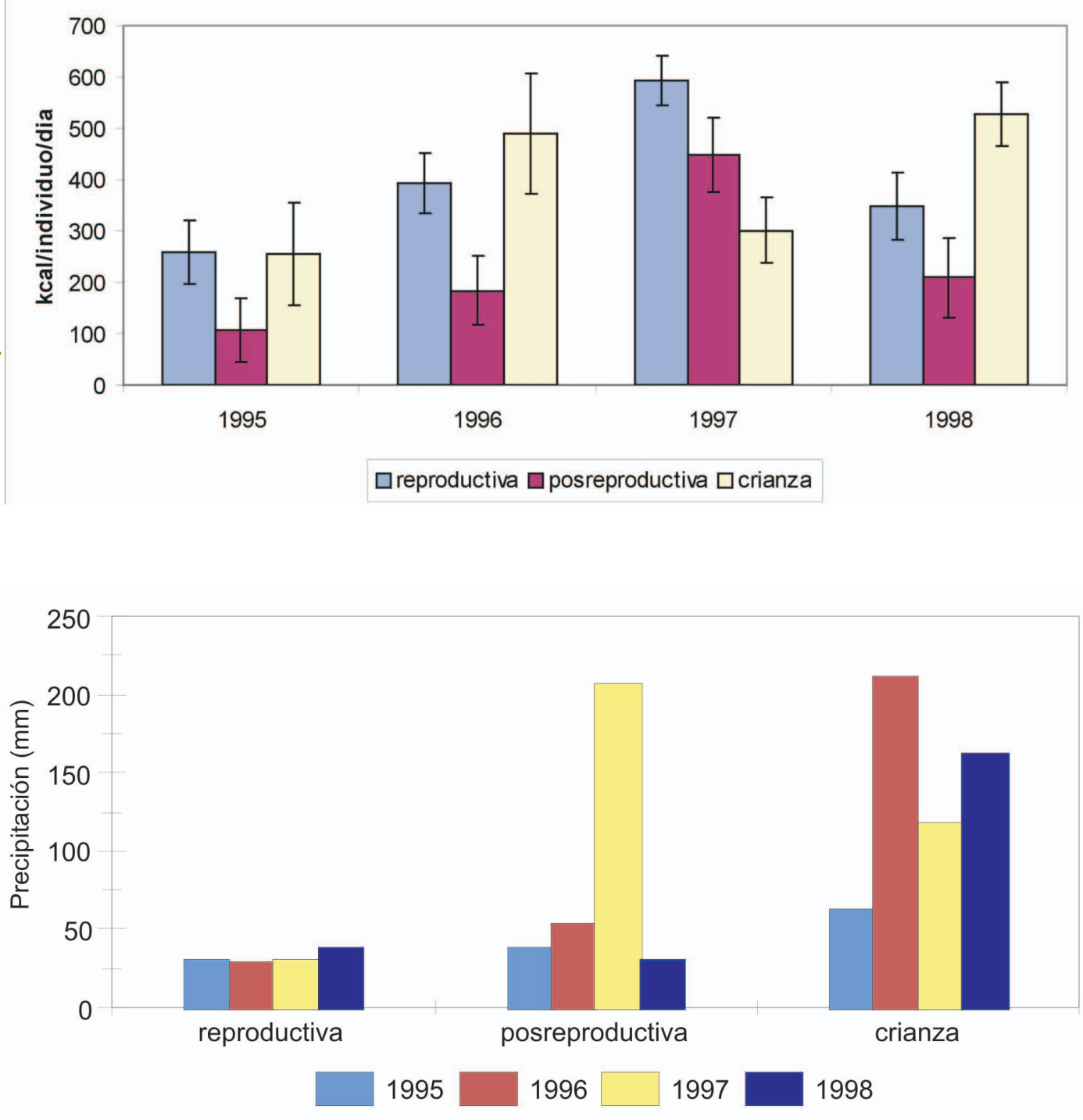

Fig. 5). En la época posreproductiva o de gestación ocurrió un cambio muy notorio en la cantidad de lluvia que cayó en 1997 durante ese período en comparación con los otros años (208 mm, CV = 102.32). En la época prereproductiva o de crianza también hubo diferencias en la precipitación en los diferentes años, siendo mayor en 1996 (212.5 $\mathrm{mm}$ ). Al hacer la correlación entre la precipitación con el gasto energético total y por pautas, los valores no fueron significativos $(\mathrm{P}>0.05)$, aunque los machos presentaron los valores más altos, en cuanto al gasto total $(r=0.46)$ y alimentación $(r=0.54)$.

\section{Discusión}

Las tres pautas de comportamiento dependen de la interacción época y año, sobre todo la de descanso o echado y la de desplazamiento que fueron altamente significativas. Esto debe estar relacionado con las condiciones del hábitat y las ambientales (temperatura y precipitación sobre todo ya que son las que van a repercutir en el comportamiento). Como ha sido demostrado en otros trabajos, cuando las temperaturas son muy elevadas 
los animales permanecerán más tiempo echados, disminuyendo su actividad, por lo cual aparentemente el gasto energético que corresponde a esta pauta será mayor en la época seca del año o en los años cuya precipitación es menor. La pauta de alimentarse si tiene cambios significativos entre las épocas ya que tanto la disponibilidad de alimento varía, como los requerimientos energéticos también de acuerdo al estado fisiológico.

Nuestros resultados muestran que el gasto energético del venado cola blanca en zonas áridas si difiere entre sexos ya que los machos gastaron más energía ( $1726 \pm 38$ kcal/ día/individuo) que las hembras (1556 35 kcal/día/individuo). Pero también se encontró que éste es menor en las zonas áridas en comparación con las zonas más norteñas como ha sido señalado por diversos autores (Lovegrove 2000; Strickland et al. 2005). Según Strickly et al. (en prensa citado por Fulbright y Ortega 2007) el requerimiento de energía para el mantenimiento de hembras no preñadas durante el invierno promedió $89 \mathrm{kcal} /$ kg de peso corporal. Esto equivaldría a 1797 kcal diarias para una hembra de $55 \mathrm{~kg}$, en contraste las hembras preñadas con un peso similar necesitaron 3191 kcal diarias (Ullrey et al. 1970). Pekins et al. (1998) encuentra que el gasto energético de los venados en descanso pero con alimento disponible fue de $1878 \mathrm{kcal}$ para una hembra de $55 \mathrm{~kg}$, mientras que en esta zona árida encontramos que fue en promedio de $800 \mathrm{kcal} / \mathrm{día} / \mathrm{ind}$. Esto comprueba que el gasto energético es efectivamente mucho menor en las zonas áridas que en las zonas más norteñas y por lo tanto, los venados responden de acuerdo a las condiciones más precarias con mecanismos tanto conductuales como fisiológicos. Los experimentos diseñados por Strickland et al. (2005) para estimar los requerimientos diarios de energía digerible para mantener la masa corporal de venados cola blanca en el sur de Texas, demostraron que éstos disminuyen cuando hay períodos extensos de restricción energética, comprobando que los venados necesitan menos requerimientos en latitudes más sureñas en comparación con las norteñas.

Los venados tuvieron un menor gasto energético en los años con condiciones de sequía más severa como fue 1995, cuando el alimento es más escaso, lo que indica que los venados en estas zonas áridas tienen una estrategia conductual para tratar de ahorrar energía cuando las condiciones ambientales son desfavorables. Es decir que optimizan el tiempo de alimentación con la mínima pérdida energética, lo que concuerda con la estrategia de forrageo óptimo (Pyke 1984). Así como en las áreas más norteñas con inviernos severos, muchos ungulados sobreviven con una baja disponibilidad de recursos alimenticios, ya que presentan mecanismos fisiológicos que permiten ahorrar calor corporal así como un pelaje con mayor aislamiento lo que contribuye a minimizar los requerimientos energéticos (Silver 1969, Strickland et al. 2005). En el caso de las zonas áridas donde la menor disponibilidad de recursos es la época seca que coincide con la época fisiológica posreproductiva, probablemente también presenten mecanismos fisiológicos que les permite minimizar los requerimientos energéticos, y de hecho se comprueba con nuestros resultados que en los años con menor precipitación y por ende más secos y con menor alimento disponible, el gasto energético fue menor. Por lo tanto, Strickland et al. (2005) demuestran que la correlación entre la restricción voluntaria en cuanto al consumo y los cambios estacionales en cantidad y calidad del alimento representa una adaptación para maximizar la sobrevivencia durante los períodos restringidos de alimento disponible. 
De hecho Arnold et al. (2004) señalan que la reducción metabólica en la producción de calor aparentemente es una reacción de los animales endotermos para sobrevivir a situaciones energéticas desafiantes. Ellos encontraron en el ciervo (Cervus elaphus) que presenta un hipometabolismo nocturno como estrategia para sobrevivir a inviernos severos, por lo cuál sugieren que en animales terrestres el hecho de disminuir el gasto energético no está restringido solo a especies que hibernan, sino que puede ser una respuesta común fisiológica a períodos de escasés de alimento y condiciones climáticas severas. Por lo tanto, podemos esperar que el venado cola blanca en zonas áridas también presente mecanismos fisiológicos que le permitan ahorrar energía. En este caso, en épocas de sequía y elevadas temperaturas, vemos que los resultados del gasto energético al comparar los diferentes años, si presentaron diferencias significativas, siendo menores en años con poca precipitación.

Además, las diferencias en cuanto a la distribución de la precipitación a lo largo del año sí es un factor que influye en el costo energético, y en la productividad de una población como fue señalado por Moen (1978), debido a que los resultados de la reproducción en cuanto a las demandas a finales del invierno y principios de la primavera, que son fijas en el tiempo (ya que sólo se reproducen una vez al año) por lo cual los venados necesitan esos requerimientos o la productividad se verá afectada. En nuestra área de estudio se encontró en otro trabajo (Soto-Werschitz et al. 2000) realizado con las hembras, que el año de 1998 cuando se atrasaron las lluvias, prácticamente la mayoría de las crías se murieron al no haber alimento suficiente para las hembras lactantes (que es el período de mayor costo energético). Sin embargo, como muestran los resultados en relación al gasto energético, parece ser que los venados ajustan esto dependiendo de las condiciones ambientales, que indudablemente no sólo repercuten en la cantidad de agua libre disponible, sino que también repercuten en la cantidad de alimento disponible, así como la cobertura de protección de insolación que les brinda la vegetación.

Como bien lo señalan Mautz et al. (1992) se necesitan más estudios para entender el metabolismo estacional de los venados ya que tienen demandas metabólicas sustancialmente más elevadas durante la primavera y verano debido tanto a cambios conductuales (en actividad y alimentación) y en procesos fisiológicos (gestación y lactancia en las hembras), y en cuanto a los machos sería la etapa de desarrollo de las astas y apareamiento.

\section{Agradecimientos}

Este estudio fue posible gracias al apoyo financiero de CONACYT a través de los proyectos No. 03270 y No. 225260-5-2480PB y al apoyo logístico de Ducks Unlimited de México. Un agradecimiento especial por su ayuda en el campo a S. Ortiz. Queremos agradecer en la toma de datos a C. Delfín Alfonso, N. D. López, C. Contreras, S. Mandujano y A. Pérez Arteaga. 
Arnold, W., T. Ruf, S. Reimoser, F. Tataruch, K. Onderscheka, y F. Schober. 2004. Nocturnal hypometabolism as an overwintering strategy of red deer (Cervus elaphus). American Journal of Physiology- Regulatory, integrative and Comparative Physiology 286:R174-R181.

Beier, P. y D. R. Mcullough. 1990. Factors influencing white-tailed deer activity patterns and habitat use. Wildlife Monographs 1-51.

BeIER, P. 1987. Sex differences in quality of white-tailed deer diets. Journal of Mammalogy 68:323-329

Bello J., S. Galuina y M. Equinua. 2001. Characterization and habitat preferences by whitetailed deer (Odocoileus virginianus) in Mexico. Journal of Range Management 54:537-545

Bello J., S. Galuina y M. Equinua. 2003a. Comparación de los movimientos del venado cola blanca en dos sitios con diferente disponibilidad de agua del Noreste de México. Pp 59- 66 in Manejo de Fauna silvestre en Amazonía y Latinoamérica. Selección de Trabajos V Congreso Internacional. (Polanco-Ochoa, R. ed.). CITES, Fundación Natura. Bogotá, Colombia.

Bello J., S. Galuina y M. Equinua. 2003b. El venado cola blanca, uso del hábitat en zonas semiáridas y con alta disponibilidad de agua del Noreste de México. Pp 67- 76 in Manejo de Fauna silvestre en Amazonía y Latinoamérica. Selección de Trabajos V Congreso Internacional. (Polanco-Ochoa, R. ed.). CITES, Fundación Natura. Bogotá, Colombia.

Bello J, S. Gallina y M. Equinua. 2004. Movements of white tailed deer and their relationship with precipitation in the northeastern of México. Interciencia 29:357-361

Fulbright, T. E. y J. A. Ortega-S. 2007. Ecología y manejo de venado cola blanca. Texas A\&M University Press. Kingsville, Texas

Gallina, S., A. Pérez-Arteaga y S. Mandujano. 1998. Patrones de actividad del venado cola blanca texano (Odocoileus virginianus texanus) en un matorral xerófilo de México. Boletín de la Sociedad Biológica. Concepción, Chile 69:221-228.

Gallina, S., P. Corona-Zárate y J. Bello. 2003. El venado cola blanca: comportamiento en zonas semiáridas del Noreste de México. Pp. 165-173 in Manejo de Fauna silvestre en Amazonía y Latinoamérica. Selección de Trabajos V Congreso Internacional. (Polanco-Ochoa, R. ed.). CITES, Fundación Natura. Bogotá, Colombia.

Gallina, S., P. Corona-Zárate y J. Bello. 2005. El comportamiento del venado cola blanca en zonas semiáridas del Noreste de México. Pp.193-203 in Contribuciones Mastozoológicas en Homenaje a Bernardo Villa. (Sánchez-Cordero, V. y R. Medellín, eds). Instituto de Biología, UNAM, Instituto de Ecología, UNAM, CONABIO.

Hudson, R. J. y R. J. Christopherson. 1985. Maintenance metabolism. Pp. 122-137 in Bionergetics of wild herbivores (Hudson, R. J., y R. G. White, eds.). CRC Press, Inc. Boca Raton, Florida.

Keyser, P. D., C. D. Guynn y H. S. Hizl. 2005. Population density-physical condition 
relationships in white-tailed deer. Journal of Wildlife Management 69:356-365.

KiE, J. G. y J. W. Thomas. 1988. Rangeland vegetation as wildlife habitat. Pp. 586-605 in Vegetation science applications for rangelands analysis and management. (Tueller, P. T. ed.). Kluwer Academic Publishers.

Krolt, J. C. 1992. A practical guide to producing and harvesting white-tailed deer. Institute of White tailed deer management and research center for applied studies in forestry. Stephen F. Austin State University. Austin, Texas.

Kroll, J. C. y B. H. Koerth. 1966. Solving the mysteries of deer movements. Center for applied Studies in Forestry. Austin state University. Nocogdoches, Texas.

Leopold, B. D. y P. R. Krausman. 1991. Factors influencing desert mule deer distribution and productivity in southwestern Texas. The Southwestern Naturalist. 36:67-74.

LoveGrove, B. G. 2000. The zoogeography of mammalian basal metabolic rate. American Naturalist 156:201-219.

Mandujano S., A. Pérez-Arteaga, R. E. Sanchez-Mantilla y S. Gallina. 1996. Diferenciación de pautas de actividad del venado con ayuda de radiotransmisores con sensor de movimiento. Acta Zoológica Mexicana (n. s.) 67:613-620.

Mautz, W. W., J. Kanter y P. J. Pekins. 1992. Seasonal metabolic rhythms of captive female white-tailed deer: a re-examination. Journal of Wildlife Management 56:656-661.

Moen, A. 1973. Wildlife Ecology. W. H. Freeman and Company. San Francisco, California

MoEN, A. 1978.Seasonal changes in heart rates, activity, metabolism, and forage intake of white-tailed deer. Journal of Wildlife Management 42:715-738,

Ockenfels, R. A. Y J. A. Bissonette. 1984. Temperature-related responses in North-Central Oklahoma white-tailed deer. Pp. 64-67 in Deer in the southwest: A workshop. (Krausman, P. R., y N. S. Smith, eds.). Arizona Cooperative wildlife research unit. University of Arizona.

Ockenfels, R. A., D. E. Brooks y C. H. Lewis. 1991. General ecology of Coues white-tailed deer in the Santa Rita Mountains. Arizona Game \& Fish Deparment Techican Report 6:1-73.

Parker, K., M. P. Gillingham, T. A. Hanley Y S. T. Robbins. 1999. Energy and Protein balance of free-ranging black-tailed deer in a natural forest environment. Wildlife Monographs 143:1-48.

Pekins, P. J., K. S. Sмith Y W. W. Mautz. 1998. The energy cost of gestation in white-tailed deer. Canadian Journal of Zoology 76:1091-1097.

PyкE, G. H. 1984. Optimal foraging theory: a critical review. Annual Review of Ecology and Systematics 15:523-575

Schwartz, C. C., M. E. Hubbert y A. W. Franzmann. 1991. Energy expenditure in moose calves. Journal of Wildlife Management 55:391-393.

Soto-Werschitz, A., S. Mandujano y S. Gallina. 2000. Comportamiento espacial de las hembras del venado cola blanca texano con y sin crías durante la época de crianza. VII Simposio sobre Venados en México. Facultad de Medicina, Veterinaria y Zootecnia-UNAM-ANGADI, México, Distrito Federal

Statsoft, INc. 2003. STATISTICA (data analysis software system) version 6. www.statsoft. COm

Strickland, B. K., D. G. Hewitt, C. A. Deyoung Y R. L. Bingham. 2005. Digestible energy 
requirements for maintenance of body mass of white-tailed deer in southern Texas. Journal of Mammalogy 86:56-60

Verme, L. J. y D. E. Ullrey. 1984. Physiology and nutrition. Pp. 91-118 in White-tailed deer: Ecology and management (Halls, L. K. ed.) Published Stackpole books.

Walımo, O. 1981. Mule and black-tailed deer of North America. A Wildlife Management Institute Book.

ZaR, J. H. 1996. Biostatistical Analysis. Prentice Hall.

Sometido: 4 octubre 2009

Revisado: 13 enero 2010

Aceptado: 3 febrero 2010

Editor asociado Jesús Maldonado 